\title{
Hyperlipidemia and Cutaneous Abnormalities in Transgenic Mice Overexpressing Human Apolipoprotein C1
}

\author{
Miek C. Jong, ${ }^{\star}$ Marion J.J. Gijbels, ${ }^{\ddagger}$ Vivian E.H. Dahlmans, ${ }^{\star}$ Patrick J.J. van Gorp, ${ }^{\ddagger}$ Sietse-Jan Koopman, ${ }^{\S}$ Maria Ponec, \\ Marten H. Hofker, ${ }^{\ddagger}$ and Louis M. Havekes* ${ }^{\star \| \star *}$ \\ *TNO-Prevention and Health, Gaubius Laboratory, 2301 CE Leiden; ${ }^{\ddagger}$ MGC-Department of Human Genetics, Leiden University, 2300

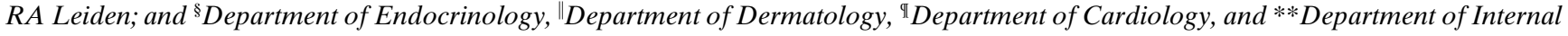 \\ Medicine, University Hospital Leiden, 2300 RC Leiden, The Netherlands
}

\begin{abstract}
Transgenic mice were generated with different levels of human apolipoprotein C1 (APOC1) expression in liver and skin. At 2 mo of age, serum levels of cholesterol, triglycerides (TG), and FFA were strongly elevated in APOC1 transgenic mice compared with wild-type mice. These elevated levels of serum cholesterol and TG were due mainly to an accumulation of VLDL particles in the circulation. In addition to hyperlipidemia, APOC1 transgenic mice developed dry and scaly skin with loss of hair, dependent on the amount of APOC1 expression in the skin. Since these skin abnormalities appeared in two independent founder lines, a mutation related to the specific insertion site of the human APOC1 gene as the cause for the phenotype can be excluded. Histopathological analysis of high expressor APOC1 transgenic mice revealed a disorder of the skin consisting of epidermal hyperplasia and hyperkeratosis, and atrophic sebaceous glands lacking sebum. In line with these results, epidermal lipid analysis showed that the relative amounts of the sebum components TG and wax diesters in the epidermis of high expressor APOC1 transgenic mice were reduced by 60 and $45 \%$, respectively. In addition to atrophic sebaceous glands, the meibomian glands were also found to be severely atrophic in APOC1 transgenic mice. High expressor APOC1 transgenic mice also exhibited diminished abdominal adipose tissue stores (a 60\% decrease compared with wild-type mice) and a complete deficiency of subcutaneous fat. These results indicate that, in addition to the previously reported inhibitory role of apoC1 on hepatic remnant uptake, overexpression of apoC1 affects lipid synthesis in the sebaceous gland and/or epidermis as well as adipose tissue formation. These APOC1 transgenic mice may serve as an interesting in vivo model for the investigation of lipid homeostasis in the skin. (J. Clin. Invest. 1998. 101:145-152.) Key words: apolipoprotein $\mathrm{C} \cdot$ sebaceous glands • sebum production $\bullet$ triglycerides $\bullet$ hypertriglyceridemia
\end{abstract}

Address correspondence to Dr. L.M. Havekes, TNO-PG, Gaubius Laboratory, P.O. Box 2215, 2301 Leiden, The Netherlands. Phone: 31-71-518-1449; FAX: 31-71-518-1904; E-mail: 1m.havekes@pg.tno.nl

Received for publication 2 June 1997 and accepted in revised form 3 November 1997.

J. Clin. Invest.

(C) The American Society for Clinical Investigation, Inc. 0021-9738/98/01/0145/08 \$2.00

Volume 101, Number 1, January 1998, 145-152

http://www.jci.org

\section{Introduction}

Apolipoprotein (apo) $\mathrm{C} 1$, a $6.6-\mathrm{kD}$ protein, is a protein constituent of triglyceride (TG) ${ }^{1}$-rich chylomicrons and VLDLs, as well as HDLs. The human APOC1 gene is located $5 \mathrm{~kb}$ downstream of the human APOE gene on chromosome 19 in the same transcriptional orientation $(1,2)$. Both genes are expressed primarily in the liver under the control of the hepatic control region element $(3,4)$, although moderate levels of APOC1 expression are also found in skin, spleen, and lung $(2,5)$.

To investigate the role of apoC1 in lipoprotein metabolism, knockout mice were generated for the APOC1 gene (6). On a high fat, high cholesterol diet, apoC1-knockout mice have elevated levels of cholesterol and delayed clearance of VLDL particles from the circulation (7). Since no effect on VLDL-TG production and lipolysis was found, the absence of apoC1 seems solely to impair the uptake of lipoprotein particles by the liver. With the generation of transgenic mice overexpressing human APOC1 in the liver, it was shown that an excess of apoC1 on the VLDL particle also impaired hepatic uptake of VLDL (8-10). Human APOC1 transgenic mice have elevated levels of both cholesterol and TG. In the absence of the LDL receptor, this inhibitory action of apoC1 on hepatic VLDL uptake was mediated via a receptor-associated protein-sensitive pathway, most likely the LDL receptor-related protein (10). Thus, since both apoC1-knockout and APOC1 transgenic mice exhibited delayed clearance of VLDL, apoC1 seems to influence the clearance of lipoprotein particles dose-dependently, but in a discontinuous way.

In addition to its influence on hepatic particle uptake, previous in vitro studies have shown that apoC1 can also activate the enzyme lecithin-cholesterol acyltransferase (11). To date, no mutations in apoC1 are known to be associated with hyperlipidemia or other clinical disorders of lipoprotein metabolism.

In this study, we report that APOC1 transgenic mice, in addition to hyperlipidemia, exhibit abnormalities of hair growth that are correlated to the level of human APOC1 gene expression in the skin. These abnormalities are associated with epidermal hyperplasia and hyperkeratosis, atrophic glands, lack of sebum, and reduced amounts of adipose tissue, which was especially apparent in the subdermal skin layer.

\section{Methods}

Animals. Transgenic mice with high expression of human APOC1 in the liver were generated as described previously (10). Two founder

1. Abbreviations used in this paper: GAPDH, glyceraldehyde-3-phosphate dehydrogenase; TG, triglyceride(s); WDE, wax diester(s); VLDLR, VLDL receptor. 
mice were characterized, and from them a low expressor strain (line 11/3) and a high expressor strain (line 11/1) were bred with C57BL/6J mice. In the breeding of male APOC1 transgenic mice (line 11/3) with female C57BL/6J mice, only female transgenic mice were generated. These findings indicate that in line 11/3, the human APOC1 gene is integrated into the $\mathrm{X}$ chromosome. In this study, females (F5 generation) were used that were hemizygous carriers $(11 / 3+/-)$ for the transgene. The males of line $11 / 3$ (F5 generation) used in this study were by definition hemizygous carriers of the APOC1 gene $(11 / 3+/ 0)$. Approximately half of the generated male 11/3 +/0 mice exhibited cutaneous abnormalities, whereas the other half appeared similar to the female $11 / 3+/-$ mice. These differences may be explained by their variable genetic background or by other factors introduced with transgene linkage to the $\mathrm{X}$ chromosome. Hemizygous males and females of line 11/1 (F5 generation) were crossbred to generate mice that were either hemizygous $(11 / 1+/-)$ or homozygous $(11 / 1+/+)$ for the transgene. In addition, hemizygous males of line 11/3 (F5 generation) were crossbred with hemizygous females of line 11/1 (F5 generation) to obtain APOC1 transgenic mice carrying copies of both line $11 / 3$ and line $11 / 1(11 / 3 \times 11 / 1)$. All mice were housed under standard conditions with free access to water and a standard mouse/rat chow diet. The microbiological status of these mice was checked regularly by routine serological, bacteriological, and histological procedures.

Serum lipid analysis. Levels of total serum cholesterol and TG (without measuring free glycerol) were determined using commercially available enzymatic kits 236691 (Boehringer Mannheim GmbH, Mannheim, Germany) and 337-B (GPO-Trinder kit; Sigma Chemical Co., St. Louis, MO). For serum FFA determination, blood samples were kept on ice and stored at $-80^{\circ} \mathrm{C}$ before measurements with an NEFA-C kit (WAKO Chemicals GmbH, Neuss, Germany), according to the manufacturer's recommendation.

For fast protein liquid chromatography fractionation, $200 \mu \mathrm{l}$ of pooled serum per group was injected onto two 25-ml Superose-6 preparation-grade columns (connected in series) (Pharmacia Biotech, Uppsala, Sweden) and eluted at a constant flow rate of $0.5 \mathrm{ml} / \mathrm{min}$ with PBS, pH 7.4. Fractions of $0.5 \mathrm{ml}$ were collected and assayed for total cholesterol and TG as described above.

Human APOC1 mRNA measurements. Total RNA was isolated from brain, heart, kidney, liver, muscle, skin, and spleen using the RNA Instapure system (Eurogentec S.A., Seraing, Belgium). RNA samples $(7.5 \mu \mathrm{g} / \mathrm{lane})$ were separated by electrophoresis through a denaturing agarose gel $(1.0 \% \mathrm{wt} / \mathrm{vol})$ containing $7.5 \%$ formaldehyde and transferred to a nylon membrane (Hybond N; Amersham Corp., Arlington Heights, IN) according to the manufacturer's recommendations. Blots were subsequently hybridized with a ${ }^{32} \mathrm{P}$-labeled probe of human APOC1 (12) at $53^{\circ} \mathrm{C}$ in a solution containing $50 \%$ formamide, and $18 \mathrm{~S}(13)$ at $65^{\circ} \mathrm{C}$ in a solution containing $0.5 \mathrm{M} \mathrm{Na}_{2} \mathrm{HPO}_{4} /$ $\mathrm{NaH}_{2} \mathrm{PO}_{4}, 1 \mathrm{mM}$ EDTA, and 7\% SDS (wt/vol).

In a different set of experiments, the amounts of human APOC1 mRNA in liver and skin of APOC1 transgenic mice were quantified with a PhosphorImager (Molecular Dynamics, Sunnyvale, CA). The amounts of human APOC1 mRNA were related to the level of glyceraldehyde-3-phosphate dehydrogenase (GAPDH) mRNA (14).

Histological analysis. Wild-type and APOC1 transgenic mice fed the chow diet were killed, and complete necroscopy including microscopic examination was performed. Tissues were fixed in $10 \%$ neutral-buffered formalin, processed, and embedded in paraffin. 3- $\mu \mathrm{m}$ sections were routinely stained with hematoxylin-phloxine-saffron.

For immunohistochemical analysis, pieces of skin tissue of wildtype and APOC1 transgenic mice were frozen in liquid nitrogen and stored at $-80^{\circ} \mathrm{C}$. Cryostat sections of $8-\mu \mathrm{m}$ thickness were fixed in acetone for $10 \mathrm{~min}$ and air-dried for at least $30 \mathrm{~min}$. After washing in $0.01 \mathrm{M}$ PBS ( $\mathrm{pH} 7.4$ ), the sections were incubated for $1 \mathrm{~h}$ with the polyclonal rabbit antisera against human apoC1, followed by a peroxidase-conjugated swine anti-rabbit $\mathrm{IgG}$ for $30 \mathrm{~min}$. After washing, the peroxidase activity was demonstrated with 3,3'-diaminobenzidine-tetrahydrochloride (Sigma Chemical Co.) in $0.5 \mathrm{mg} / \mathrm{ml}$ Tris- $\mathrm{HCl}$ buffer ( $\mathrm{pH} 7.6$ ) containing $0.01 \% \mathrm{H}_{2} \mathrm{O}_{2}$.
Hyperinsulinemic, euglycemic clamp study. Fasted mice were anesthetized with an intraperitoneal injection of sodium pentobarbital (30 $\mu \mathrm{g} / \mathrm{g}$ body wt, Nembutal; Sanofi Sante b.v., Maassluis, The Netherlands), and the right jugular vein was cannulated. Body temperature was maintained with the use of a warming pad. The protocol for measurement of insulin-stimulated glucose uptake was as described previously (15). Briefly, insulin was infused for $120 \mathrm{~min}$ at a constant rate of $6 \mathrm{mU} / \mathrm{kg} \cdot \mathrm{min}$, and a variable $25 \% \mathrm{D}$-glucose solution was infused to maintain plasma glucose levels at $7 \mathrm{mmol} / \mathrm{liter} .\left[3-{ }^{3} \mathrm{H}\right]$ glucose $(0.1 \mu \mathrm{Ci} / \mathrm{min}$; Amersham Corp.) was infused throughout the experiment to determine the glucose turnover rate (15). Blood samples were collected from the tail tip, and steady state glucose turnover was determined from 90 to $120 \mathrm{~min}$.

Isolation and lipid analysis of mouse epidermis. Wild-type and APOC1 transgenic mice fed the chow diet were killed, and the dorsal skin was dissected. To separate the epidermis from the underlying tissue, skin preparations were heated for $1 \mathrm{~min}$ at $60^{\circ} \mathrm{C}$. Epidermal lipids were extracted using the method of Bligh and Dyer (16), dissolved in chloroform/methanol 2:1 ( $\mathrm{vol} / \mathrm{vol})$, and stored at $-20^{\circ} \mathrm{C}$ under nitrogen until use. The extracted lipids were separated by one-dimensional high performance TLC as described previously (17), and standards (Sigma Chemical Co.) were run in parallel. The quantification was performed after charring using a photodensitometer with automatic peak integration (Desaga, Heidelberg, Germany).

\section{Results}

Characterization of APOC1 transgenic mice. Two founder mice carrying the human APOC1 construct (lines 11/3 and 11/1) were generated and partly characterized as described previously (10). In this study, the effect of APOC1 expression levels was examined in closer detail. As described in Methods, mice of line 11/3 showed X-linked transgene transmission. APOC1 transgenic mice of line 11/1 showed autosomal transmission. Through breeding of APOC1 transgenic 11/1 +/- male and female mice, wild-type (34\%), hemizygous $11 / 1+/-(47 \%)$, and homozygous mice $(11 / 1+/+)(19 \%)$ were obtained (an offspring of 79 mice). The hemi- and homozygous 11/1 mice were identified through quantitative Southern blot analysis using liver DNA. In addition, crossbreeding of $11 / 3+/ 0$ males with $11 / 1+/-$ females generated an offspring (21 mice) which consisted of wild-type males (38\%), 11/3 females (19\%), 11/1 males $(19 \%)$, and $11 / 3 \times 11 / 1$ females $(24 \%)$. The latter offspring group did not include wild-type females, $11 / 3$ males, or $11 / 1 \mathrm{fe-}$ males, due to the X-linked transgene transmission of line 11/3.

Northern blot analysis of a series of different tissues from APOC1 transgenic mice of line 11/3 demonstrated that human APOC1 mRNA expression in these mice was confined mainly to the liver, although low but significant levels of expression were also found in other tissues (Fig. 1). A similar distribution of human APOC1 mRNA was found in mice of line 11/1 (results not shown). The APOC1 transgenic mice of the various strains displayed different levels of human APOC1 mRNA in the liver and in the skin (Fig. 2). More specifically, as quantified with a PhosphorImager, 11/1 +/- mice had 1.7-fold elevated levels of human APOC1 mRNA in the liver compared with $11 / 3+/-$ mice (Table I). No further significant elevation in APOC1 expression was observed in the livers of $11 / 3 \times 11 / 1$, male $11 / 3+/ 0$, and 11/1 +/+ mice (Table I). Human APOC1 mRNA levels in the skin were elevated 1.3-fold in $11 / 1+/-$ mice compared with $11 / 3+/-$ mice. However, in contrast to APOC1 mRNA levels in the liver, APOC1 expression in the skin was further elevated in $11 / 3 \times 11 / 1,11 / 3+/ 0$, and $11 / 1+/+$ mice (approximately three- to fourfold compared with $11 / 3+/-$ 


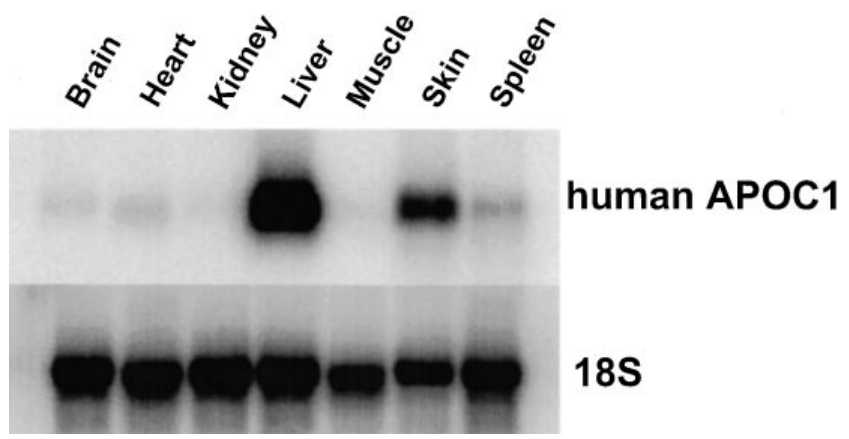

Figure 1. Tissue transgene expression pattern in APOC1 transgenic mice. Total RNA was isolated from brain, heart, kidney, liver, muscle, skin, and spleen of APOC1 transgenic mice $(11 / 3+/-)$ using the RNA Instapure system. $7.5 \mu \mathrm{g}$ RNA was used for Northern blot analysis followed by hybridization with a probe for human APOC1 cDNA (top) and $18 \mathrm{~S}$ (bottom).

mice) (Table I). This gene dose-dependent increase in human APOC1 mRNA levels in the skin of APOC1 transgenic mice was reflected by the amount of the human apoC1 protein in the dermis as shown by immunohistochemical analysis using polyclonal antibodies against human apoC1 (results not shown).

Serum lipid levels in APOC1 transgenic and wild-type mice. Table I summarizes the serum lipid levels in fasted APOC1 transgenic and wild-type mice on a chow diet. Mice of line $11 / 3+/-$ showed slightly elevated levels of serum cholesterol, TG, and FFA compared with wild-type mice. In mice of line 11/1(11/1 +/-), serum lipid levels were further elevated. In addition, $11 / 3 \times 11 / 1,11 / 3+/ 0$, and $11 / 1+/+$ mice showed strongly elevated levels of serum cholesterol, TG, and FFA on a normal chow diet (Table I). As determined by fast performance liquid chromatography analysis, all of the serum cholesterol in wild-type mice was confined to the HDL-size particles, whereas the elevated serum cholesterol and TG levels in APOC1 transgenic mice was due mainly to an accumulation of VLDLsize particles (results not shown).

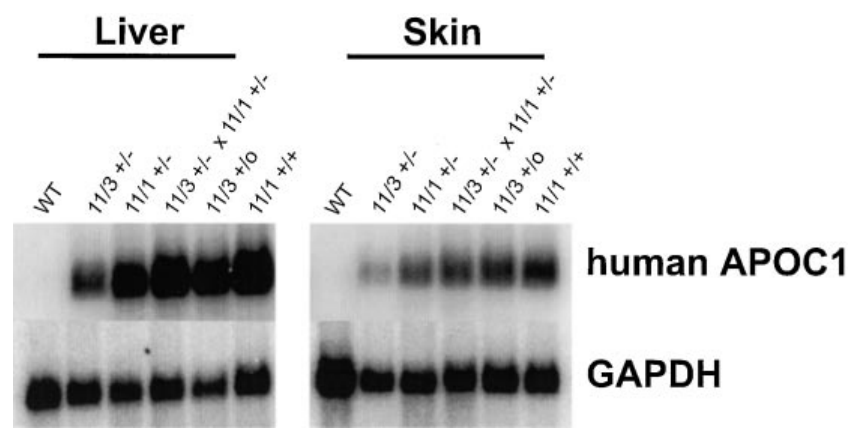

Figure 2. Northern blot analysis of livers and skin from APOC1 transgenic mice. Total RNA was isolated from the liver (left) and skin (right) of wild-type and APOC1 transgenic mice using the RNA Instapure system. Human APOC1 transcripts were detected using a probe of human APOC1 cDNA (12) (top) and a rat GAPDH cDNA (13) (bottom) as a reference.

To investigate whether the elevated levels of TG and FFA in the homozygous APOC1 transgenic mice were associated with insulin resistance, the glucose turnover rate in $11 / 1+/+$ and wild-type mice was measured using the hyperinsulinemic clamp. As shown in Table I, 11/1 +/+ mice showed an increased glucose turnover rate, as measured under conditions of physiological hyperinsulinemia, compared with wild-type mice. These results indicate that insulin resistance does not develop in APOC1 transgenic mice with severe hypertriglyceridemia.

Gross pathology and histological examination of APOC1 transgenic and wild-type mice. Macroscopically, APOC1 transgenic mice with high levels of APOC1 expression in their skin $(11 / 3 \times 11 / 1,11 / 3+/ 0$, and 11/1 +/+; Table I) exhibited cutaneous abnormalities. These abnormalities started at a young age, with dry skin, fine epidermal scaling, and hair loss, which became more severe upon aging. The hair loss was apparent throughout the whole skin area. In addition, these mice developed extensive crusts around the eyes and pruritus, often caus-

Table I. Human APOC1 mRNA Levels, Serum Lipid Levels, and Whole Body Glucose Uptake in APOC1 Transgenic and Wild-type Mice

\begin{tabular}{|c|c|c|c|c|c|c|c|}
\hline \multirow[b]{2}{*}{ Mice } & \multirow[b]{2}{*}{ Gender } & \multicolumn{2}{|c|}{ APOC1 mRNA } & \multicolumn{3}{|c|}{ Serum lipids } & \multirow[b]{2}{*}{ Glucose uptake } \\
\hline & & Liver & Skin & $\mathrm{TC}$ & TG & FFA & \\
\hline & & $\%$ & $\%$ & mmol/liter & mmol/liter & mmol/liter & $\mathrm{mg} / \mathrm{kg} \cdot \min$ \\
\hline Wild-type & Female & ND & ND & $1.8 \pm 0.2$ & $0.3 \pm 0.2$ & $1.3 \pm 0.3$ & $36.2 \pm 3.5$ \\
\hline $11 / 3+/-$ & Female & $100 \pm 36$ & $100 \pm 47$ & $2.4 \pm 0.5^{\ddagger}$ & $0.8 \pm 0.3^{\ddagger}$ & $1.8 \pm 0.9$ & \\
\hline $11 / 1+/-$ & Female & $169 \pm 25 *$ & $133 \pm 34$ & $5.2 \pm 1.0^{\ddagger}$ & $4.6 \pm 1.7^{\ddagger}$ & $2.8 \pm 0.9^{\ddagger}$ & \\
\hline $11 / 3+/-\times 11 / 1+/-$ & Female & $177 \pm 13^{*}$ & $295 \pm 78^{* \S}$ & $8.0 \pm 1.3^{\ddagger}$ & $7.3 \pm 1.2^{\ddagger}$ & $3.9 \pm 0.3^{\ddagger}$ & \\
\hline $11 / 3+/ 0$ & Male & $170 \pm 30 *$ & $398 \pm 109 * \S$ & $10.5 \pm 1.7^{\ddagger}$ & $14.3 \pm 5.2^{\ddagger}$ & $3.2 \pm 0.4^{\ddagger}$ & \\
\hline $11 / 1+/+$ & Female & $188 \pm 22 *$ & $292 \pm 22 * \S$ & $20.4 \pm 7.4^{\ddagger}$ & $21.4 \pm 9.3^{\ddagger}$ & $5.5 \pm 1.5^{\ddagger}$ & $55.5 \pm 5.3^{\ddagger}$ \\
\hline
\end{tabular}

Liver, skin, and serum were collected from fasted wild-type mice and the respective APOC1 transgenic mice. All mice were 2 mo old, and fed a regular chow diet. Human APOC1 mRNA concentrations are relative to an internal standard GAPDH, and are expressed as a percentage of 11/3+/mice. Total cholesterol (TC), TG, FFA, and whole body glucose uptake were measured as described in Methods. Values are expressed as the mean of at least five mice per group \pm SD for mRNA and serum lipid measurements, and three mice per group for glucose turnover studies. $N D$, Not detectable. ${ }^{\circledR}$ Transgenic mice exhibiting cutaneous abnormalities. ${ }^{*} P<0.05$, the difference between $11 / 3+/-$ mice and other APOC 1 transgenic lines. ${ }^{\ddagger} P<$ 0.05, the difference between APOC1 transgenic and wild-type mice using the nonparametric Mann-Whitney $U$ test. 


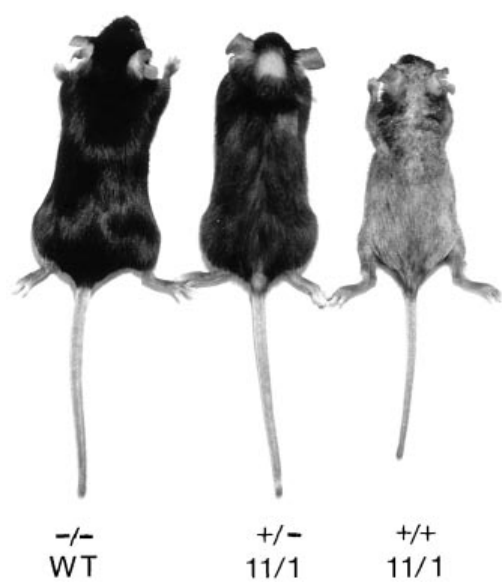

Figure 3. Gross pathology in APOC1 transgenic mice at 2 mo of age. Mice hemizygous for the APOC1 gene $(11 / 1+/-)$ have a thin hair-coat compared with wild-type mice $(W T,-/-)$. Mice homozygous for the APOC1 gene $(11 / 1+/+)$ appear almost hairless and exhibit thickened dry and scaly skin.

ing self-inflicted wounds which necessitated killing before the age of 3 mo (according to institutional guidelines concerning ethics of experimentation). Gross examination revealed that the cutaneous abnormalities in APOC1 transgenic mice described above were also apparent in mice kept under specificpathogen-free breeding conditions. Since APOC1 transgenic $11 / 3 \times 11 / 1,11 / 3+/ 0$, and 11/1 +/+ mice all exhibited identical

Table II. Tissue Weight of APOC1 Transgenic and Wild-type Mice

\begin{tabular}{lcc}
\hline \multicolumn{1}{c}{ Tissues } & Wild-type & $11 / 1+/+$ \\
\hline & $g$ & $g$ \\
Total body weight & $24.73 \pm 1.06$ & $25.52 \pm 2.12$ \\
Abdominal adipose tissue & $0.92 \pm 0.24$ & $0.38 \pm 0.06^{*}$ \\
Liver & $1.53 \pm 0.09$ & $2.35 \pm 0.27^{*}$ \\
Heart & $0.16 \pm 0.03$ & $0.23 \pm 0.04^{*}$ \\
Spleen & $0.13 \pm 0.02$ & $0.29 \pm 0.03^{*}$ \\
& & \\
\hline
\end{tabular}

Tissues from female $11 / 1+/+$ and female wild-type mice (2.5 mo old) were dissected and weighed. All mice were fed a regular chow diet. Values are expressed as the mean $\pm \mathrm{SD}$ of six mice per group. $* P<0.05$, the difference between $11 / 1+/+$ and wild-type mice using the nonparametric Mann-Whitney $U$ Test.

cutaneous abnormalities on gross appearance, further characterization and analysis of skin tissues were performed on wildtype and APOC1 transgenic mice of strain 11/1.

As presented in Fig. 3, hemizygous APOC1 transgenic mice displayed a thinner hair coat compared with wild-type mice, whereas homozygous carriers of the human APOC1
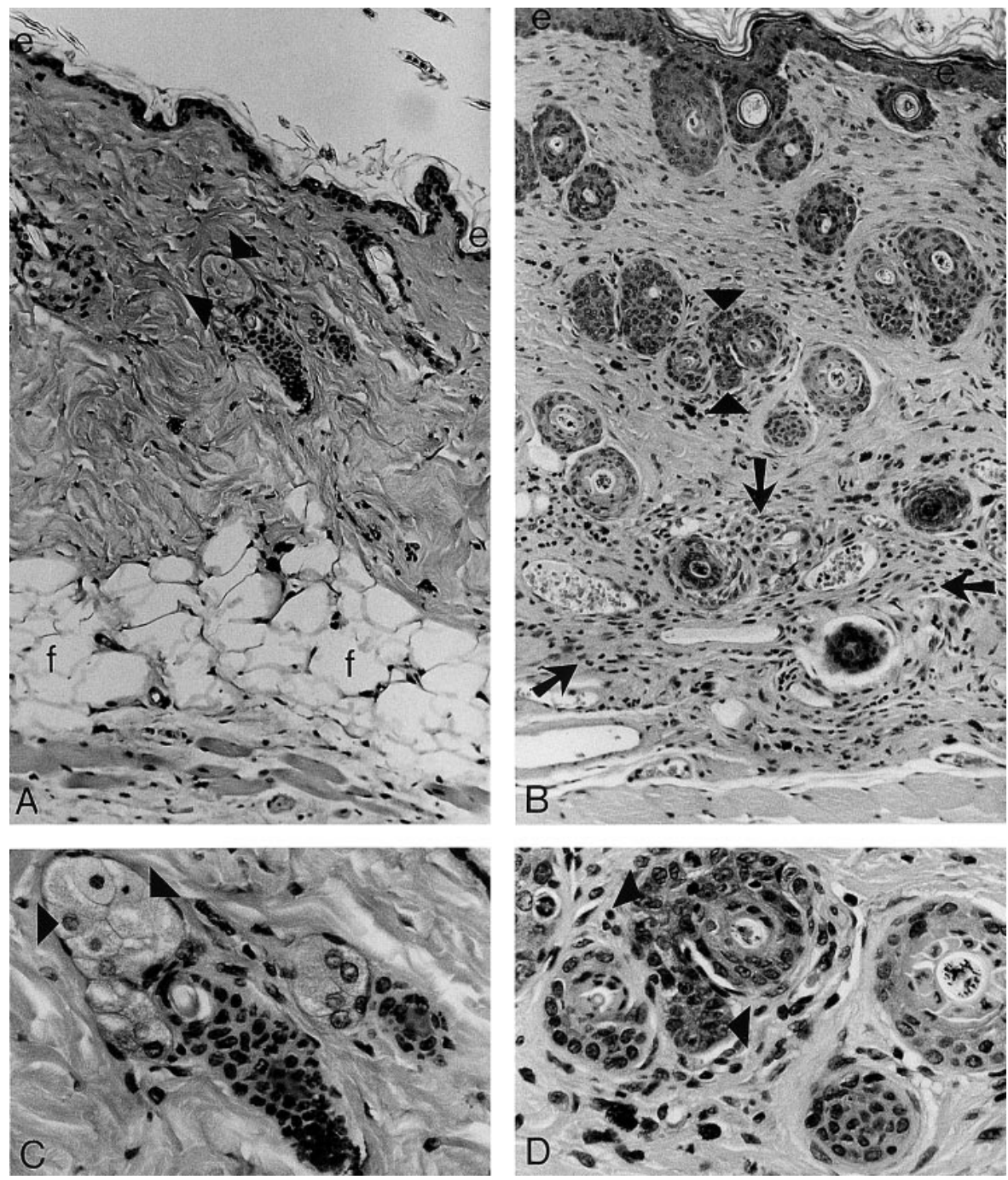

Figure 4. Representative photographs of skin lesions observed in APOC1 transgenic mice. $(A)$ Skin of a wild-type mouse; note the epidermis $(e)$ and sebaceous glands (arrowheads). $f$, Fat cells in subdermal skin layer; $\times 220$. (B) Skin of a homozygous $11 / 1$ $+/+$ APOC1 transgenic mouse; note the thickened epidermis $(e)$, atrophic sebaceous glands (arrowheads), and inflammatory cells in the dermis (arrows); $\times 220$. (C) Higher magnification $(\times 515)$ of the sebaceous gland in the wild-type skin; note the foamy appearance of the cytoplasm (arrowheads). (D) Higher magnification $(\times 515)$ of the atrophic sebaceous gland in APOC1 transgenic mice $(11 / 1+/+)$. Note that the foamy appearance of the cytoplasm has disappeared (arrowheads). 
transgene appeared almost hairless. Furthermore, homozygous APOC1 transgenic mice appeared smaller than their hemizygous and wild-type littermates (Fig. 3). However, the body weights as determined at $2.5 \mathrm{mo}$ of age were similar for homozygous APOC1 transgenic and wild-type mice (Table II). Several tissues were dissected from female homozygous APOC1 transgenic and wild-type mice and weighed. Remarkably, abdominal fat pads were decreased in the APOC1 transgenic mice compared with wild-type mice (Table II). In addition, APOC1 transgenic mice exhibited an enlarged liver, heart, and spleen (Table II).

On histological examination, atrophic sebaceous glands were observed in the skin of the severely affected APOC1 transgenic mice $(11 / 1+/+)$ (compare Fig. $4 A$ for wild-type with Fig. $4 B$ for APOC1 transgenic mice; arrowheads indicate the sebaceous glands). Similar observations were made in the skins of $11 / 3 \times 11 / 1$ and $11 / 3+/ 0$ mice, and to a lesser extent in $11 / 1+/-$ mice (not shown). Fig. $4 C$ shows a higher magnification of the sebaceous glands in wild-type mice, with the foamy appearance of the cytoplasm that indicates the presence of lipid droplets (sebum). In contrast, at higher magnification, the cytoplasm of the atrophic sebaceous glands in APOC1 transgenic mice had lost its foamy appearance completely (Fig. $4 D$ ).
The epidermis of the affected APOC1 transgenic mice was considerably thicker compared with the epidermis of wild-type mice (compare Fig. $4 A$ with Fig. $4 B$ ). Remarkably, the skin of the affected APOC1 transgenic mice lacked subcutaneous adipose tissue, in contrast to the presence of many large fat cells in the subcutis of wild-type mice (compare Fig. $4 A$ with $4 B ; f$ indicates the fat cells in wild-type skin). Furthermore, homozygous APOC1 transgenic mice exhibited skin lesions that varied from a mild folliculitis to a mild mixed cellular infiltration in the dermis (Fig. 4 B; arrows indicate cellular infiltration of predominantly macrophages, granulocytes, and mast cells). At older age, these lesions developed into a severe dermatitis, with a moderate epidermal hyperplasia with hyper- and parakeratosis. The epidermis was necrotic with extensive infiltration of inflammatory cells (macrophages and granulocytes) in the dermis (not shown).

The modified sebaceous glands of the eyelids, the so-called meibomian glands, were also severely atrophic in the APOC1 transgenic animals (compare Fig. $5 A$ for wild-type with Fig. $5 B$ for APOC1 transgenic mice; arrowheads indicate meibomian glands). As shown in Fig. $5 C$, at higher magnification, the cytoplasm of the meibomian glands of wild-type mice appeared foamy due to the presence of meibum. The meibomian
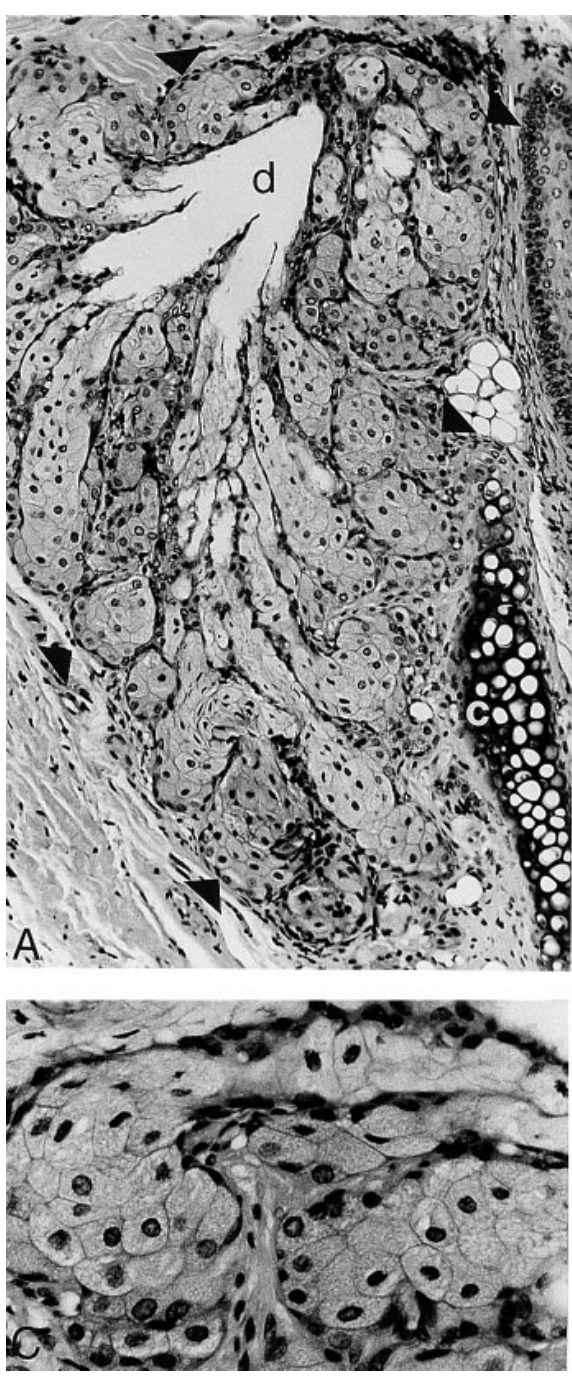
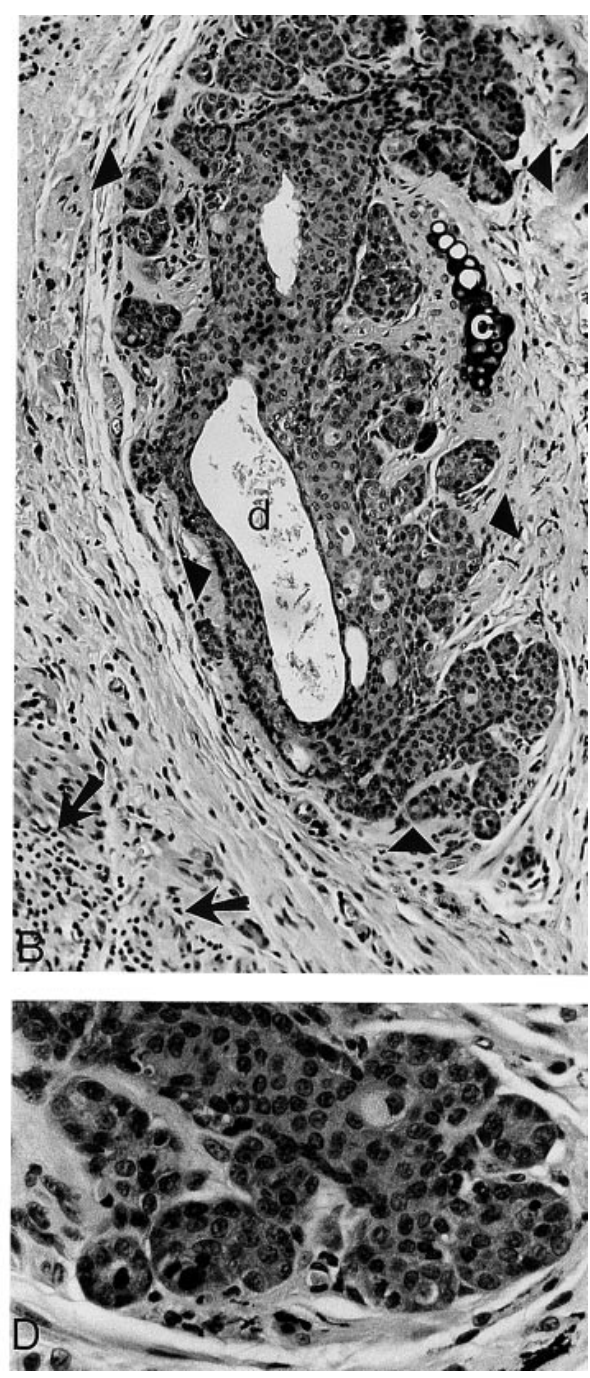

Figure 5. Representative photographs of the atrophic meibomian gland in APOC1 transgenic mice. $(A)$ Meibomian gland (arrowheads) in a wild-type mouse. $d$, Intralobular duct; $c$, cartilage; $\times 220$. (B) Meibomian gland in an APOC1 transgenic mouse $(11 / 1+/+)$; note the atrophic meibomian gland (arrowheads) and inflammatory cells (arrows) in the outer surface of the eyelid. $d$, Intralobular duct; $c$, cartilage; $\times 220$. (C) Higher magnification $(\times 515)$ of the meibomian gland in wild-type mice; note the foamy appearance of the cytoplasm. $(D)$ Higher magnification $(\times 515)$ of the atrophic meibomian gland in APOC1 transgenic mice $(11 / 1+/+)$; note that the foamy appearance of the cytoplasm has disappeared. 
glands of APOC1 transgenic mice lacked this foamy appearance (Fig. 5 D). Furthermore, no inflammation was observed around the meibomian glands of APOC1 transgenic mice. However, the outer surface of the eyelids showed sebaceous gland atrophy and cellular infiltrations of inflammatory cells similar to those described for the skin (Fig. 5 B; arrows indicate cellular infiltration of inflammatory cells).

The male preputial gland, another modified sebaceous gland in mice, was not affected in APOC1 transgenic mice (not shown).

Lipid composition of APOC1 transgenic and wild-type mouse epidermis. Lipids were extracted from the epidermis of wild-type and APOC1 transgenic mice and analyzed by high performance TLC. Polar epidermal lipids, including phospholipids, cholesterol sulfate, and glucosphingolipids, were 8-20\% of the total epidermal lipids (by weight). The epidermal lipids also contained different ceramides $(5-10 \%$ of total lipid, by weight), but the largest amount consisted of nonpolar (neutral) lipids, including TG, sterols, wax diesters (WDE), and FFA (70$87 \%$ of total lipid, by weight). No major differences were found in the polar lipids or ceramides upon TLC analysis between wild-type and APOC1 transgenic mice (not shown). The analysis of the neutral lipids in the mouse epidermis of APOC1 transgenic and wild-type mice is presented in Table III. The relative TG and WDE contents of epidermal lipids from APOC1 transgenic mice $(11 / 1+/-)$ were strongly reduced compared with wild-type mice. The relative proportions of FFA and free cholesterol were increased, and the lanosterol proportions were decreased, in the epidermis of APOC1 transgenic mice. No differences were observed in the relative cholesterol ester content. In the homozygous APOC1 transgenic mice $(11 / 1+/+)$, similar changes in epidermal lipids were found, but they were more profound than in hemizygous $11 / 1+/-$ mice (Table III).

\section{Discussion}

Transgenic mice overexpressing human APOC1 have elevated levels of serum cholesterol and TG due to an accumulation of VLDL remnants in their circulation (8-10). Since the extrahepatic lipolysis rate of VLDL-TG is not inhibited, an impaired uptake of VLDL remnants by the liver is considered to be the primary metabolic defect in APOC1 transgenic mice (10).

The extent of the hyperlipidemia in APOC1 transgenic mice was related to the level of transgene expression in the liver $(9,10)$. In this study, we examined the effect of APOC1 expression levels in greater detail and observed that in one transgenic mouse line (line 11/3), the human APOC1 gene was linked to the $\mathrm{X}$ chromosome. Due to random inactivation of one of their X chromosomes, female $11 / 3+/-$ mice were expected to carry only half the copies of the transgene in the active state compared with $11 / 3$ male mice. As shown in Table I, female $11 / 3+/-$ mice indeed exhibited lower levels of human APOC1 mRNA in the liver than males of line 11/3 (Table I). Surprising however, was the observation that hepatic human APOC1 mRNA levels in homozygous $11 / 1+/+$ mice and $11 / 3 \times$ 11/1 mice were not further elevated compared with 11/1 +/mice (Table I). These data strongly suggest that overexpression of human APOC1 in mice may be subjected to feedback regulation, which can prevent excessive levels of human APOC1 mRNA in the liver by reducing transcription rates or mRNA stability.

APOC1 transgenic mice also displayed human APOC1 expression in the skin, although to a much lower extent than in the liver (Fig. 1). The observation that the hyperlipidemia and human APOC1 expression in the skin were further elevated in $11 / 1+/+$ and $11 / 3 \times 11 / 1$ mice compared with hemizygous $11 / 1$ $+/-$ mice (Table I) suggests that the presence of human apoC1 in the skin may contribute to the elevated serum lipid levels in APOC1 transgenic mice. Whether apoC1 originating in the skin may actually be transported into the blood circulation, to give rise to a further inhibition of remnant uptake and, subsequently, hyperlipidemia, is unknown at the present time.

In addition to hyperlipidemia, high expressor APOC1 transgenic mice (Table I) exhibited cutaneous abnormalities that included hair loss at a young age, epidermal hyperplasia/hyperkeratosis, and atrophic sebaceous glands. Since these cutaneous abnormalities appeared in two independent founder lines (line $11 / 3$ and line 11/1), with different integration sites of the human APOC1 gene, it can be excluded that the observed skin abnormalities in APOC1 transgenic mice are due to aberrant expression related to the specific transgene insertion site. In addition, it was demonstrated that the cutaneous abnormalities were clearly associated with the level of human APOC1 expression in the skin and became apparent in all mice carrying copies of both line 11/3 and line 11/1 (11/3 × 11/1 mice).

On histological examination, it appeared that the atrophic sebaceous glands in the skin of the high expressor APOC1 transgenic mice lacked sebum (Fig. $4 B$ ). These findings were confirmed by the analysis of mouse epidermal lipids showing that the relative TG and WDE contents, which are reported to be the major components of sebum $(18,19)$, were strongly reduced in the epidermis of the high expressor APOC1 transgenic mice (Table III). Since sebum is suggested to $(a)$ prevent

Table III. Neutral Lipid Composition of the Mouse Epidermis

\begin{tabular}{llccrr}
\hline \multicolumn{1}{c}{ Mice } & TG & WDE & FFA & FC & CE \\
\hline \multicolumn{5}{c}{ LAN } \\
Wild-type & $32.9 \pm 6.5$ & $29.9 \pm 6.6$ & $3.0 \pm 0.4$ & $6.4 \pm 1.8$ & $10.2 \pm 2.2$ \\
$11 / 1+/-$ & $15.4 \pm 5.8^{*}$ & $21.9 \pm 2.2$ & $8.0 \pm 2.4^{*}$ & $13.9 \pm 3.1^{*}$ & $10.8 \pm 0.8$ \\
$11 / 1+/+$ & $12.6 \pm 2.9^{*}$ & $16.6 \pm 1.7^{*}$ & $10.2 \pm 0.9^{*}$ & $14.8 \pm 1.1^{*}$ & $11.9 \pm 2.5$ \\
& & & & & $0.4 \pm 0.1^{*}$
\end{tabular}

Skins were dissected from female wild-type and female hemizygous (+/-) and homozygous $(+/+)$ APOC1 transgenic mice of strain 11/1. All mice were 2 mo old, and fed a regular chow diet. Epidermal TG, WDE, FFA, free cholesterol $(F C)$, cholesterol ester $(C E)$, and lanosterol $(L A N)$ were measured by high performance TLC as described in Methods. Values are the mean \pm SD of three mice per group. $* P<0.05$, the difference between APOC1 transgenic and wild-type mice using the nonparametric Mann-Whitney $U$ test. 
bacteria from persisting in the hair canal and $(b)$ prevent water loss from the skin (18), scaly and dry skin with excessive hair loss and infiltrations of inflammatory cells may be caused by the absence of sebaceous outflow of sebum onto the skin of these mice.

APOC1 transgenic mice also showed atrophic meibomian glands. The meibomian glands are found in the border of the eyelid and secrete meibum that is somewhat similar to sebum (20). It has been reported that under normal conditions, meibum forms a lipid layer around the eyes to protect them from excessive dehydration (21). Thus, in high expressor APOC1 transgenic mice, lack of meibum may cause eye dehydration, reflected by the extensive crusts observed around their eyelids.

The mechanism behind the development of cutaneous abnormalities in high expressor APOC1 transgenic mice could be that apoC1 disturbs lipid synthesis in the sebaceous glands and/or the epidermis. Previous studies in humans have shown that the amount of lipid derived from the blood circulation may become rate-limiting for the synthesis of sebum lipid components in the skin $(22,23)$. In these studies, it was reported that within a few days after commencing a fast, the incorporation of FFA into sebum components such as TG and WDE was reduced by $50 \%$. Thus, from this point of view, it is tempting to speculate that the high amounts of apoC1 present in the serum or skin of APOC1 transgenic mice may prevent FFA transport from the circulation to the skin tissue, leading to poor sebum production (Table III). In agreement with such a hypothesis is the finding that APOC1 transgenic mice exhibit strongly elevated levels of serum FFA (Table I). Since, after sampling, serum fractions of mice were stored immediately on ice, these elevated FFA levels are most likely due to overexpression of APOC1 rather than to spontaneous hydrolysis of their high serum TG after collection of the blood sample. Furthermore, it has been shown that the glucose turnover rate measured with the hyperinsulinemic clamp is increased in APOC1 transgenic mice compared with control mice (Table I). According to the Randle cycle (24), this increased glucose turnover rate in APOC1 transgenic mice indicates that the FFA oxidation is reduced in these mice.

Further observations suggesting an interference of apoC1 with FFA transport to tissues were made through gross and histological examinations showing that the affected APOC1 transgenic mice exhibited reduced amounts of adipose tissue in the abdomen (Table II) and developed no adipose tissue in the subdermal skin layer. Similar findings have been reported recently in lipoprotein lipase-deficient mice by Weinstock et al. (25). During their short life span of about $18 \mathrm{~h}$, lipoprotein lipase-deficient pups failed to develop normal amounts of adipose tissue, which was especially apparent in the subdermal skin layer. Furthermore, mice deficient for the VLDL receptor (VLDLR) have also been reported to exhibit reduced amounts of adipose tissue (26). These findings are in line with the hypothesis that the VLDLR may act as a docking protein for efficient TG-rich lipoprotein lipolysis and subsequent delivery of FFA into muscles and fat cells $(27,28)$. Previous studies have shown that apoC1-enriched lipoprotein particles are defective in binding to the VLDLR in vivo (29). Whether the impaired binding of APOC1 transgenic lipoproteins to the VLDLR may contribute to the elevated serum FFA levels and reduced amounts of abdominal adipose tissue in APOC1 transgenic mice is currently under investigation.

The abnormalities observed in high expressor APOC1 transgenic mice are somewhat similar to those seen in the mutant mouse asebia ( $a b)(30-32)$. It was suggested that the pathological changes in the $a b$ mice are caused by an inherited defect in the metabolism of branched-chain fatty acids, reflected by the presence of crystal-filled macrophages (composed of unusual branched-chain fatty acids) in their dermis (33). However, the APOC1 transgenic mice described in our study clearly lack these crystals in macrophages. Furthermore, the observations that $(a)$ the $a b$ mutation is located on mouse chromosome 19 (34), whereas the mouse APOC1 gene is located on chromosome 7 , and $(b)$ only mice that are homozygous for the $a b$ mutation develop the specific asebian characteristics, whereas in APOC1 transgenic mice, the cutaneous abnormalities are dependent on the levels of APOC1 expression, make it highly unlikely that the abnormalities described for the $a b$ mice are due to defects in apoC1 or vice versa.

The hyperkeratosis and epidermal hyperplasia in high expressor APOC1 transgenic mice are similar to those observed in a variety of dermatopathies, including human psoriasis. However, unlike psoriasis, APOC1 transgenic mice have atrophic sebaceous glands and an acute dermal inflammation. Furthermore, the high expressor APOC1 transgenic mice share several characteristics with a very complex and rare syndrome in humans, the AREDYLD syndrome $(35,36)$. Briefly, patients with this syndrome display dry skin, abnormalities of hair growth, a generalized deficiency of subcutaneous fat, and several other dental, nail, and skeleton anomalies. However, unlike AREDYLD syndrome subjects $(35,36)$, APOC1 transgenic mice do not develop insulin resistance (Table I).

In summary, APOC1 transgenic mice develop hyperlipidemia and skin abnormalities dependent on the levels of human APOC1 expression. These abnormalities are characterized by epidermal hyperplasia and hyperkeratosis, atrophic sebaceous/meibomian glands, reduced amounts of sebum and meibum in the skin, and a reduction in adipose tissue mass. Although a direct experimental link with an analogous human disease remains to be established, these mice may provide a useful animal model for studying the barrier function and lipid homeostasis in the skin.

\section{Acknowledgments}

We are grateful to Arij Weerheim for excellent technical assistance.

This research was supported by the Netherlands Heart Foundation, the Netherlands Foundation of Scientific Research (projects 901-04-092 and 903-39-117), and by a grant of BIOMED 2 from the specific Research Technology Development Program of the European Commission (BMH4-CT96-0898). M. Hofker is an Established Investigator of the Netherlands Heart Foundation.

\section{References}

48. Smit, M., E. van der Kooij-Meijs, R.R. Frants, L.M. Havekes, and E.C Klasen. 1988. Apolipoprotein gene cluster on chromosome 19. Definite localization of the APOC2 gene and the polymorphic Hpa I site associated with type III hyperlipoproteinemia. Hum. Genet. 78:90-93.

49. Lauer, S.J., D. Walker, N.A. Elshourbagy, C.A. Reardon, B. Levy-Wilson, and J.M. Taylor. 1988. Two copies of the human apolipoprotein C1 gene are linked closely to the apolipoprotein E gene. J. Biol. Chem. 263:7277-7286.

50. Shachter, N.S., Y. Zhu, A. Walsh, J.L. Breslow, and J.D. Smith. 1993. Localization of a liver-specific enhancer in the apolipoprotein E/C-I/C-II gene locus. J. Lipid Res. 34:1699-1707.

51. Simonet, W.S., N. Bucay, S.J. Lauer, and J.M. Taylor. 1993. A far-downstream hepatocyte-specific control region directs expression of the linked human apolipoprotein E and $\mathrm{C} 1$ genes in transgenic mice. J. Biol. Chem. 268: 8221-8229. 
52. Simonet, W.S., N. Bucay, R.E. Pitas, S.J. Lauer, and J.M. Taylor. 1991. Multiple tissue-specific elements control the apolipoprotein E/C-II gene locus in transgenic mice. J. Biol. Chem. 266:8651-8654.

53. van Ree, J.H., M.H. Hofker, W.J.A.A. van den Broek, J.M.A. van Deursen, H. van der Boom, R.R. Frants, B. Wieringa, and L.M. Havekes. 1995. Increased response to cholesterol feeding in apolipoprotein $\mathrm{C} 1$-deficient mice. Biochem. J. 305:905-911.

54. Jong, M.C., J.H. van Ree, V.E.H. Dahlmans, R.R. Frants, M.H. Hofker, and L.M. Havekes. 1996. Reduced very low density lipoprotein fractional catabolic rate in apolipoprotein C1-deficient mice. Biochem. J. 321:445-450.

55. Jong, M.C., V.E.H. Dahlmans, P.J.J. van Gorp, M.L. Breuer, M.J.T.M. Mol, A. van der Zee, R.R. Frants, M.H. Hofker, and L.M. Havekes. 1996. Both lipolysis and hepatic uptake of VLDL are impaired in transgenic mice coexpressing human apolipoprotein E*3Leiden and human apolipoprotein $\mathrm{C} 1$. Arterioscler. Thromb. Vasc. Biol. 16:934-940.

56. Shachter, N.S., T. Ebara, R. Ramakrishnan, G. Steiner, J.L. Breslow, H.N. Ginsberg, and J.D. Smith. 1996. Combined hyperlipidemia in transgenic mice overexpressing human apolipoprotein C1. J. Clin. Invest. 98:846-855.

57. Jong, M.C., V.E.H. Dahlmans, P.J.J. van Gorp, K. Willems van Dijk, M.L. Breuer, M.H. Hofker, and L.M. Havekes. 1996. In the absence of the low density lipoprotein receptor, human apolipoprotein $\mathrm{C} 1$ overexpression in transgenic mice inhibits the hepatic uptake of very low density lipoproteins via a receptor-associated protein-sensitive pathway. J. Clin. Invest. 98:2259-2267.

58. Soutar, A.K., C.W. Garner, H.N. Baker, J.T. Sparrow, R.L. Jackson, A.M. Gotto, Jr, and L.C. Smith. 1975. Effect of the human plasma apolipoproteins and phosphatidylcholine acyl donor on the activity of lecithin:cholesterol acyltransferase. Biochemistry. 14:3057-3064.

59. Zannis, V.I., J. McPherson, G. Goldberger, S.U. Karathanasis, and J.L. Breslow. 1984. Synthesis, intracellular processing, and signal peptide of human apolipoprotein E. J. Biol. Chem. 259:5495-5499.

60. Church, G.M., and W. Gilbert. 1984. Genome sequencing with chain terminating inhibitors. Proc. Natl. Acad. Sci. USA. 81:1991-1995.

61. Fort, P.I., L. Marty, M. Piechaczyk, S.E. Sabrouty, C. Dani, P. Jeanteur, and J.M. Blanchard. 1985. Various rat adult tissues express only one major mRNA species from the glyceraldehyde-3-phosphate-dehydrogenase multigenic family. Nucleic Acids Res. 13:1431-1442.

62. Koopmans, S.J., S.F. de Boer, H.C.M. Sips, J.K. Radder, M. Frölich, and H.M.J. Krans. 1991. Whole body and hepatic insulin action in normal, starved and diabetic rats. Am. J. Physiol. 260:825-832.

63. Bligh, E.G., and W.J. Dyer. 1959. A rapid method for total lipid extraction and purification. Can. J. Biochem. Physiol. 37:911-917.

64. Ponec, M., A. Weerheim, J. Kempenaar, A.M. Momaas, and D.H. Nugteren. 1988. Lipid composition of cultured human keratinocytes in relation to their differentiation. J. Lipid Res. 29:949-961.

65. Stewart, M.E., and D.T. Downing. 1991. Chemistry and function of mammalian sebaceous lipids. Adv. Lipid Res. 24:263-301.

66. Wilkinson, D.I., and M.A. Karasek. 1966. Skin lipids of a normal and a mutant (asebic) mouse strain. J. Investis. Dermatol. 47:449-455.

67. Tiffany, J.M. 1987. The lipid secretion of the meibomian glands. $A d v$. Lipid Res. 22:1-62.
68. Nicolaides, N., and E.C. Santos. 1985. The di- and triesters of the lipids of steer and human meibomian glands. Lipids. 20:454-467.

69. Pochi, P.E., D.T. Downing, and J.S. Strauss. 1970. Sebaceous gland response in man to prolonged total caloric deprivation. J. Investis. Dermatol. 55: 303-309.

70. Downing, D.T., J.S. Strauss, and P.E. Pochi. 1972. Changes in skin surface lipid composition induced by severe caloric restriction in man. Am. J. Clin. Nutr. 25:365-367.

71. Randle, P.J., P.B. Garland, and C.N. Hales. 1963. The glucose fatty acid cycle. Its role in insulin sensitivity and the metabolic disturbances in diabetus mellitus. Lancet. 1:785-789.

72. Weinstock, P.H., C.L. Bisgaier, K. Aalto-Setälä, H. Radner, R. Ramakrishnan, S. Levak-Frank, A.D. Essenburg, R. Zechner, and J.L. Breslow. 1996. Severe hypertriglyceridemia, reduced high density lipoprotein, and neonatal death in lipoprotein lipase knockout mice. J. Clin. Invest. 96:2555-2568.

73. Frykman, P.K., M.S. Brown, T. Yamamoto, J.L. Goldstein, and J. Herz. 1995. Normal plasma lipoproteins and fertility in gene-targeted mice homozygous for a disruption in the gene encoding very low density lipoprotein receptor. Proc. Natl. Acad. Sci. USA. 92:8453-8457.

74. Yamamoto, T., S. Takahashi, J. Sakai, and Y. Kawarabayasi. 1993. The very low density lipoprotein receptor. Trends Cardiovasc. Med. 3:144-148.

75. Yamamoto, T., A. Hoshino, S. Takahashi, Y. Kawarabayasi, H. Iijima, and J. Sakai. 1995. The role of the very low density lipoprotein receptor in the metabolism of plasma lipoproteins containing ApoE. Ann. NY Acad. Sci. 748: $217-225$.

76. Jong, M.C., V.E.H. Dahlmans, P.J.J. van Gorp, K. Willems van Dijk, S.-J. Koopmans, L. Chan, M.H. Hofker, and L.M. Havekes. 1996. The binding of VLDL to the VLDL receptor is inhibited by an excess of apoC1. Cir. Suppl. 94: 4081. (Abstr.)

77. Gates, A.H., and M.A. Karasek. 1965. Hereditary absence of sebaceous glands in the mouse. Science. 148:1471-1473.

78. Wilkinson, D.I., and M.A. Karasek. 1966. Skin lipids of a normal and a mutant (asebic) mouse strain. J. Investis. Dermatol. 47:449-455.

79. Gates, A.H., F.D. Arundell, and M.A. Karasek. 1969. Hereditary defect of the pilosebaceous unit in a new double mutant mouse. J. Investis. Dermatol. 52:115-118.

80. Brown, W.R., and M.H. Hardy. 1988. A hypothesis on the cause of chronic epidermal hyperproliferation in asebia mice. Clin. Exp. Dermatol. 13: 74-77.

81. Green, M.C. 1981. Catalog of mutant genes and polymorphic loci. In Genetic Variants and Strains of the Laboratory Mouse. M.C. Green, editor, Fisher, New York. 16.

82. Pinheiro, M., N. Freire-Maia, E.A. Chautard-Freire-Maia, L.M.B. Araujo, and B. Liberman. 1983. AREDYLD: a syndrome combining an acrorenal field defect, ectodermal dysplasia, lipoatrophic diabetes and other manifestations. Am. J. Med. Genet. 16:29-33.

83. Breslau-Siderius, E.J., J. Toonstra, J.A. Baart, H.P.F. Koppeschaar, J.A. Maassen, and F.A. Beemer. 1992. Ectodermal dysplasia, lipoatrophy, diabetes mellitus, and amastia: a second case of the AREDYLD syndrome. Am. J. Med. Genet. 44:374-377. 